\title{
Long Distance Pronominalisation and Global Focus
}

\author{
Janet Hitzeman and Massimo Poesio \\ CSTR and HCRC, University of Edinburgh \\ 2 Buccleuch Place, Edinburgh EH8 9LW, Scotland \\ $\{$ J.Hitzeman, Massimo.Poesio\}eed.ac.uk
}

\begin{abstract}
Our corpus of descriptive text contains a significant number of long-distance pronominal references ( $8.4 \%$ of the total). In order to account for how these pronouns are interpreted, we re-examine Grosz and Sidner's theory of the attentional state, and in particular the use of the global focus to supplement centering theory. Our corpus evidence concerning these long-distance pronominal references, as well as studies of the use of descriptions, proper names and ambiguous uses of pronouns, lead us to conclude that a discourse focus stack mechanism of the type proposed by Sidner is essential to account for the use of these referring expressions. We suggest revising the Grosz \& Sidner framework by allowing for the possibility that an entity in a focus space may have special status.
\end{abstract}

\section{Motivation}

We call LONG-DISTANCE PRONOMINALISATIONS those cases of pronoun use in which the antecedent of the pronoun does not occur either in the same sentence as the pronoun or in the immediately preceding one, but further back in the text. These cases are thought to be rare on the basis of studies such as (Hobbs, 1978), which found that $98 \%$ of pronoun antecedents in the corpus analysed were in the same sentence as the pronoun or the previous one. However, our analysis of a small corpus of oral descriptions of museum items collected for the ILEX project (Hitzeman et al., 1997) revealed that longdistance pronouns are much more common in this kind of data -four times as common, in fact: out of a total of 83 pronouns, $7(8.4 \%)$ were long-distance. The typical pattern of long-distance pronominalisation in the ILEX dialogues is shown in (1), where the pronoun him in the last sentence refers to the jeweller, mentioned most recently two sentences earlier.

\begin{abstract}
JO: Okay, thank you. Shall we look at the object in case number 16 , number 1 ? There's a set of three objects here.

LG: 1. Yes.

2. What these symbolise for me are one of the preoccupations of the 1980 s, which is recycling.

3. The jeweller who made these bangles was particularly interested in the idea of using intrinsically worthless material-material that had been thrown away, old junk-and he lavished on those materials an incredibly painstaking and time-consuming technique, so that the amount of time put into the labour of making these jewels bears absolutely no relation to the value of the materials that he's used.

4. And if you look at, for instance, the bangle at the bottom- that's the blue and red one- what looks as though it's painted decoration is in fact inlaid; it's bits of cut-off razor-blade, biro, knitting needles, inlaid into layer after layer of resin. which is done in emulation of Japanese lacquer technique.

5. And that particular bangle took him something like 120 hours of work.
\end{abstract}

All 7 long-distance pronouns in the ILEX dialogues we have studied refer to discourse entities introduced in background text in this way.

Unlike Sidner's theory of focus (Sidner, 1979), the theory of the attentional state in (Grosz and Sidner, 1986) (henceforth: G\&S) does not include explicit provision for long-distance pronominalisations, although some of the necessary tools are potentially already there, as we will see. The component of the theory that deals with pronominal reference, centering theory (Grosz et al., 1995), only accounts for cases in which the antecedent of a pronoun is introduced by the previous sentence; cases such as (1) have to be handled by different mechanisms. In this paper we look the phenomenon of long-distance pronominalisation in some detail, examining data from different domains, and consider 
its implications for G\&S's theory.

\section{Theories of focus}

Space unfortunately prevents a full discussion of Grosz's (1977), Sidner's (1979), and G\&S's (1986) theories of focus and the attentional state in this abstract. The crucial aspects of these theories, for the purpose of the discussion below, are as follows. First of all, G\&S propose a distinction between two components of the attentional state: the GLOBAL FOCUS, structured as a stack of focus spaces and accessed to interpret definite descriptions; and the LOCAL FOCUS, consisting of the information preferentially used to interpret pronouns. In addition, they adopt CENTERING THEORY (Grosz et al., 1995) as a theory of the local focus.

Secondly, although G\&S's theory integrates ideas from both Grosz's and Sidner's original theories, and although both of these theories assumed a stack structure, the global focus in G\&S's theory is structured as a stack of focus spaces, as in Grosz's original proposal, rather than as a stack of discourse foci, as in Sidner's original theory. The claim that different parts of the attentional state are accessed when resolving pronouns and definite descriptions is supported, broadly speaking, by psycholinguistic research (see, e.g., (Garrod, 1993)). The main claims of centering theory are also consistent with psycholinguistic results (Hudson, 1988; Gordon et al., 1993). To our knowledge, however, the choice of a stack of focus spaces over a stack of discourse foci has never been motivated; yet this decision plays a crucial role in our problem, as we will see.

A point worth keeping in mind throughout the following discussion is that, although the concept of $\mathrm{CB}$ (centering theory's name for the current most salient entity) was originally introduced as 'roughly corresponding to Sidner's concept of discourse focus', in fact it is not clear that the two concepts are capturing the same intuitions (Poesio and Stevenson, 1998). Although it is often the case that the $C B$ and the discourse focus coincide, this is not true in general.' For the purposes of this paper, however, we will assume that the two notions do coincide, and will use the neutral term MOST SALIENT ENTITY (MSE) to refer to the discourse focus / CB of a particular segment of text.

'This intuitive impression was confirmed by a recent study (Giouli. 1996), whose author tracked both the 'intuitive CB' and the 'intuitive discourse focus' of 8 Map Task conversations.

\section{The Data}

The Intelligent Labelling Explorer (ILEX) project is building a system that generates descriptions of objects displayed in a museum gallery. ${ }^{2}$ In order to generate the most natural descriptions of the objects, dialogues with a museum curator were collected, describing objects in the National Museum of Scotland's 20th Century Jewellery Gallery. We will refer to this corpus as the ILEX corpus. In the dialogues, the curator (LG) moves from case to case as directed by an observer (JO) and describes the jewels in each case, as in example (1).

The work described here is part of two related projects: SOLE, the goal of which is to extend the ILEX system with the capability of generating prosodically adequate speech, and GNOME, which is concerned with the generation of nominal expressions. A second corpus of museum object descriptions was collected for use with SOLE; we will refer to this corpus as the SOLE corpus.

\section{Analysis}

\subsection{First Hypothesis}

Because G\&S's theory of the attentional state already hypothesises global focus structures in addition to the local attentional structures assumed in centering theory, the simplest explanation for our cases of long-distance pronominalisation is to hypothesise that readers exploit the global focus to resolve pronouns in such cases.

Assuming that the global focus is involved in these cases, instead of complicating the local focus/centering theory, is consistent with the little available psychological evidence -e.g., with the results of Clark and Sengul (1979), who observed a slowdown in reading times for the sentence containing the pronoun when the antecedent is not in the same or the previous sentence, implying that long-distance pronominal anaphora are handled differently.

Furthermore, suggesting that these pronouns are resolved by accessing the global focus would not really amount to a revision of the basic assumptions of G\&S's theory. Although no explicit proposal concerning the respective roles of local focus and global focus in pronoun resolution has ever been made in the literature on the G\&S framework, cases of pronouns involving access to the global attentional structure rather than to the local focus have

\footnotetext{
${ }^{2}$ http: / /www.cogsci.ed.ac.uk/alik/ilex/ systemintro.html
} 
already been discussed in this literature. So-called RETURN-POPS, which are pronouns that signal a return to a superordinate discourse segment, were discussed by Grosz (1977) and then in detail in (Fox, 1987). In (2), for example, sentence 5 resumes the segment interrupted by $2-4$; the antecedent for the pronoun her is supposed to be found on the stack, although the details of this process have never really been discussed. ${ }^{3}$

1. C: Ok Harry. I'm have a problem that uh my-with today's economy my daughter is norking,

2. H: I missed your name.

3. C: Hank.

4. H: Go ahead Hank.

5. C: as well as her husband

A second case of pronouns that clearly seem to involve access to some global structure are so-called 'generic' pronouns, such as they in (3):

John went back to the hotel. He looked for Mary in their room, but couldn't find her. They told him that she had left about an hour earlier.

(We are not aware of any account of these uses of pronouns within the G\&S framework.)

As we will see in a moment, the long-distance pronouns observed in the ILEX dialogues are neither generic pronouns nor return-pops; nevertheless, we are going to show that these cases, as well, are resolved by accessing the global focus.

\subsection{Long-distance pronouns need not be return-pops}

The use of him in the last sentence of (1) could only be termed a RETURN-POP if it were to involve a return to the previous discourse segment which 'pops over' sentence 4 (And if you look at, for instance, the bangle at the bottom ... ) and 'closes off' the material introduced in that sentence. But this is clearly not the case, as shown by the fact that the final sentence contains a reference to both the jeweller and the bangle. Indeed, the bangle could also be referred to with a pronoun: And it took him something like 120 hours of work. The fact that pronouns and definite NPS in the last sentence can refer back to material in the 4th sentence indicates that this material must still be on the stack.

\footnotetext{
'This example is from (Pollack et al., 1982).
}

\subsection{Discourse Structure in the Example Text}

Before discussing how the global focus is used for resolving pronouns such as the long-distance pronoun in the last sentence of (1), we need to discuss the structure of these examples: i.e., is the part of (1) which has the jeweller as MSE (2nd sentence) still on the stack when the part that describes details of the jewel and contains the long-distance pronoun (3rd and 4th sentence) is processed?

Answering this question is made more difficult by the fact that G\&S's theory of the intentional structure is very abstract, and therefore does not help much in specific cases, especially when the genre is not task-oriented conversations. More specific indications concerning the structure of the relevant example, and more in general of the conversations in the ILEX corpus, are given by Rhetorical Structure Theory (RST) (Mann and Thompson, 1988), ${ }^{4}$ although even with RST it is still possible to analyse any given text in many different ways. Nevertheless, we believe that the structure depicted in Figure 1 is a plausible analysis for (1); an alternative analysis would be to take the 4th and 5th sentence as elaborations of and he lavished on those materials an incredibly painstaking technique ... , but in this case, as well (and in all other rhetorical structures we could consider) sentences 4 and 5 are satellites of sentence 3. (We have employed the set of rhetorical relations currently used to analyse the ILEX data.)

The relation between G\&S's and RST's notion of structure has been analysed by, among others, (Moore and Paris, 1993; Moser and Moore, 1996). According to Moser and Moore, the relation can be characterised as follows: an RST nucleus expresses an intention $I_{n}$; a satellite expresses an intention $I_{s}$; and $I_{n}$ dominates $I_{s}$. Thus, in (1), the nucleus of the exemplification relation, sentence 3 , would dominate the satellite, consisting of sentences 4 and 5 . We will make the same assumption here. Hence we can assume that the third sentence in (1) will still be on the stack when processing the 4 th and 5 th sentences. This would also hold for the alternative rhetorical structures we have considered. ${ }^{5}$

\footnotetext{
${ }^{4}$ Fox, as well, used RST to analyse the structure of texts in her study of the effect of discourse structure on anaphora (Fox, 1987).

${ }^{5}$ Some readers might wonder whether it wouldn't be simpler to assume that all of the utterances in (1) are part of the same segment. This assumption would indeed make the antecedent accessible; however, it would not explain the data, not at least if we assume that it is centering theory that determines
} 


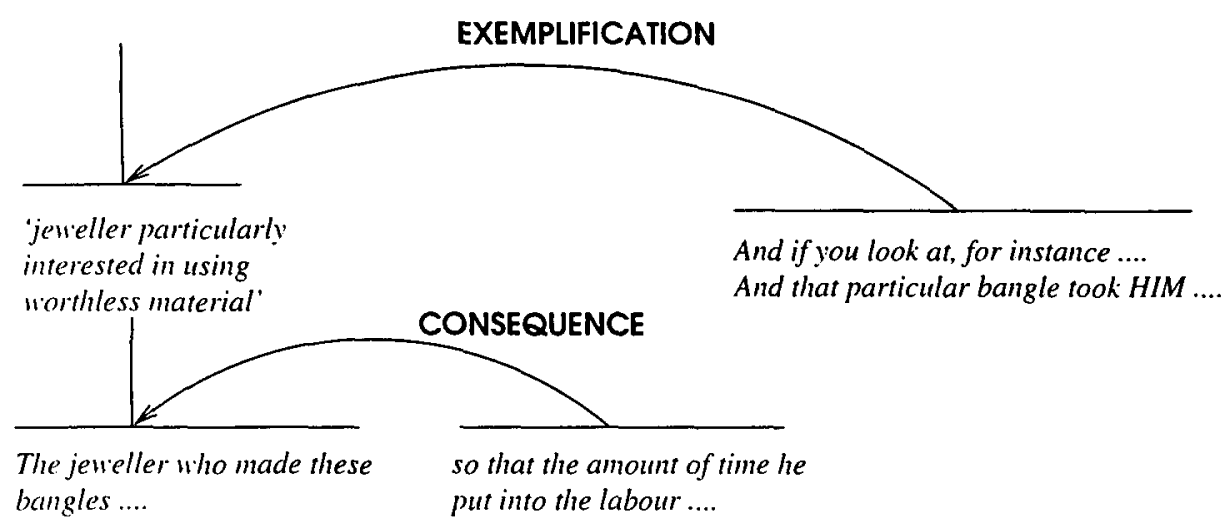

Figure 1: A possible analysis of (1).

\subsection{What Goes on the Stack?}

We can finally turn to the task of explaining how the global focus is used to resolve long-distance pronominalisations. The simplest explanation consistent with the G\&S's framework would be to assume that resolving such pronouns involves searching for the first discourse entity in the focus space stack that satisfies gender and number constraints. Under the assumptions about the discourse structure of examples like (1) just discussed, this explanation would indeed account for that example; there is evidence, however, that additional constraints are involved. The first bit of evidence is that the presence on the focus space stack of an appropriate antecedent does not always make the use of a long distance pronoun felicitous. Consider the following fragment of an article that appeared in The Guardian, January 28, 1995, p.3.

Joan Partington, aged 44, from Bolton, Lancashire, has six children. The eldest are two 17-year-old twin boys, one awaiting a heart bypass operation and the other with severe behavioral problems. A 13-year-old son has hydrocephalus. She was living with her husband when Wigan magistrates ordered her to be jailed unless she paid $f 5$ per week, although he earned only $f 70$ per week as a part-time postman.

anaphoric reference, since centering does not explain how a pronoun can refer to an antecedent two sentences back. Assuming that there is more than one segment in such texts, instead, will turn out to be not just a more plausible assumption about segmentation; it will also give us a simple way to explain the data.
The use of he in the last sentence is awkward, even though there is a discourse entity on the focus space stack - the husband- that would satisfy the constraints imposed by the pronoun. This seems to indicate that the elements of a focus space are not all equally accessible.

The second relevant bit of evidence concerns the use of proper names in the ILEX corpus. It may happen in the ILEX dialogues that a designer like Jessie King is first mentioned by name in a segment where she is not the main topic of discussion, as in Other jewels in the Bohemian style include a brooch by Jessie King. If this is the case, then when later we're talking about another jewel that King designed, she will have to be introduced again with a full proper name, Jessie King, rather than simply King. If, however, she becomes the 'main topic' of discussion, then later, whenever we talk about her again, we can use reduced forms of her proper name, such as King. Again, this difference is not easy to explain in terms of focus spaces if we assume that all objects in a focus space have the same status.

A third class of expressions providing evidence relevant to this discussion are bridging descriptions, i.e., definite descriptions like the door that refer to an object associated with a previously mentioned discourse entity such as the house, rather than to the entity itself (Clark, 1977). Poesio et al. (1997; 1998) report experiments in which different types of lexical knowledge sources are used to resolve bridging descriptions and other cases of definite descriptions that require more than simple string match for their resolution. Their results indicate that to resolve bridging descriptions it is not sufficient simply to find which of the entities in the current focus 
space is semantically closest to the bridging description: in about half of the cases of bridging descriptions that could be resolved on the basis of the lexical knowledge used in these experiments, the focus spaces contained an entity whose description was more closely related to that of the bridging description than the one of the actual antecedent(s).

This evidence about infelicitous pronouns, proper names, and bridging descriptions suggests that the entities in a focus space are not all equally salient. In fact, one could even wonder if we need focus spaces at all; i.e., if Sidner's original proposal - according to which it's just the MSE that goes on the stack, not the whole focus space - is correct. A revision of G\&S's theory along these lines-i.e., in which the focus space stack is replaced by an MSE stack- would still explain (1), since the jeweller is clearly the MSE of sentence 3 ; indeed, all 7 cases of long-distance pronouns found in the ILEX corpus have a previous MSE as their antecedent. But, in addition, this revision would explain the awkwardness of (4): the husband was never an MSE, so it would not be on the stack.

A global focus of this type would also give us a way to formulate a restriction on using shortened forms of proper names that would account for the facts observed in the ILEX corpus: reduced NPS are allowed for entities that have been introduced as MSEs, full NPS are needed otherwise. And finally, keeping track of previous MSEs seems essential for bridging descriptions as well: in order to find the reasons for the low performance of algorithms for resolving bridging descriptions entirely based on lexical knowledge, (Poesio et al., 1998) examined the bridging descriptions their corpus to find out their 'preferred' antecedent. ${ }^{6}$ They found that the preferred antecedent of a bridging description is a previous MSE in 54 out of 203 cases. In the SOLE corpus, 8 out of 11 bridging descriptions relate to the MSE.

Does this mean, then, that we can get rid of focus spaces, and assume that it's MSEs that go on the stack? Before looking at the data, we have to be clear as to what would count as evidence one way or the other. Even an approach in which only previous MSES are on the stack would still allow access to entities which are part of what Grosz called the IMPLICIT FOCUS of these MSEs, i.e., the entities that

\footnotetext{
"As discussed in (Poesio and Vieira, 1998), in general there is more than one potential 'antecedent' for a bridging description in a text.
}

are 'strongly associated' with the MSEs. This notion of 'strong association' is difficult to define- in fact, it is likely to be a matter of degree- but nevertheless it is plausible to assume that the objects 'strongly associated' with a discourse entity A do not include every discourse entity $B$ which is part of a situation described in the text in which $\mathrm{A}$ is also involved; and this can be tested with linguistic examples, up to a point. For example, whereas definite descriptions like the radiator cap can easily be resolved in a null context to a car, descriptions like the dog can't, as shown by the infelicity of $(5 \mathrm{~d})$ as a continuation of (5b), even though dogs in cars are not uncommon; some contextual antecedent is needed.

$$
\begin{aligned}
& \text { a. Mary saw a dark car go by quickly. } \\
& \text { b. It was a bright, warm day. } \\
& \text { c. The radiator cap was shining in the sun. } \\
& \text { d. The dog was enjoying the warmth. }
\end{aligned}
$$

The question we have to answer, then, is whether the only information that is available as part of the attentional state is what is 'strongly associated' with one of the previous MSES, or, instead, all of the information mentioned in the text. ${ }^{\text {? }}$

Now, sentences like (5a) license both bridging descriptions to the car, as in (5c), and to Mary, as in Her hat had become very hot. Whatever we take the MSE of (5a) to be, it seems implausible to argue that both the bridging descriptions the radiator cap and Her hat are resolved by looking at the objects 'strongly associated' with that discourse entity. It is much simpler to assume that both Mary and the car are still accessible as part of the focus space constructed to represent the situation described by the text. This also holds for what we have called 'generic' pronouns, as shown by (3), in which they refers to individuals associated with the hotel mentioned in the first sentence, not to the MSE, John.

And indeed, Sidner assumed two stacks- one of discourse foci, the other of actor foci. But even this extension would not be enough, because the antecedent of a bridging description is not always an entity explicitly introduced in the text, but can also be a more abstract DISCOURSE TOPIC, by which we

\footnotetext{
${ }^{7}$ Notice however that the claim that only MSEs go on the stack does not entail that everything else in the text is simply forgotten- the claim is simply that that information is not available for resolving references anymore; presumably it would be stored somewhere in 'long term memory'. Conversely, the claim that everything stays on the stack would have to be supplemented by some story concerning how information gets forgotten-e.g., by some caching mechanism such as the one proposed by Walker (1996).
} 
mean an issue / proposition that can be said to characterise the content of the focus space as a whole. In a corpus analysis done in connection with (Poesio et al., 1997; Poesio et al., 1998), we found that 7 out of 70 inferential descriptions were of this type; in the SOLE corpus, in which 3 out of 11 bridging descriptions behave this way. An example of this use is the description the problem below, that refers to the problem introduced by the first sentence in the text:

Solo woodwind players have to be creative if they want to work a lot, because their repertoire and audience appeal are limited. ... The oboist Heinz Holliger has taken a hard line about the problem ...

Reference to abstract objects in general seem to require maintaining information about the events and situations described by a text on the stack-see, e.g., (Webber, 1991). So, it looks like what we need is something of a compromise between the notion of global focus implicit in Sidner's original proposal and that proposed by G\&S.

\subsection{The proposal}

The following hypothesis about the global focus and its use in pronoun resolution seems to provide the best account of the evidence we have examined:

1. The global focus consists of a stack of focus spaces, as in G\&S's proposal. Each of these focus spaces can be summarised as being 'about' some object / proposition / issueindeed, more than one-for which we will use the term DISCOURSE TOPICS; but, in addition,

2. Each focus space may be optionally associated with a MOST SALIENT ENTITY (MSE) explicitly introduced in the text.

3. The antecedent for a non-generic pronoun is preferentially to be found in the local focus; if none is available, one of the MSEs associated with a focus space on the stack can also serve as antecedent. ${ }^{8}$

4. Generic pronouns refer back to the situation described by the current focus space;

5. Bridging descriptions can be related either to an entity in the current focus space, or to an MSE, or to a discourse topic;

${ }^{x}$ This would explain the difference in reading times obscrved by (Clark and Sengul, 1979).
6. Definite descriptions can refer back to any entity in the global focus, including discourse topics.

The reason for using the term 'optional' in 2 is that whereas focus spaces can always be described as being about something, they are not always associated with a 'most salient entity': e.g., the first sentence in (6) introduces several topics (woodwind players, their need to be creative, etc.) but does not introduce an MSE.

\section{Related Work}

In a recent paper, Hahn and Strube (1997) propose to extend centering theory with what is, essentially, Sidner's stack of discourse foci, although their algorithm for identifying the $\mathrm{CP}$ is not identical to Sidner's. Their analysis of German texts shows a rather good performance for their algorithm, but, as only MSES are predicted to be accessible, none of the anaphors depending on focus space information could be resolved. Their algorithm also appears to treat definite descriptions and pronouns uniformly as 'anaphors', which seems problematic in the light of psychological evidence showing that they behave differently, and examples like the following:

$$
\begin{aligned}
& \text { a. John } \\
& \text { b. Saw Mary. He } \mathrm{Jehn}_{i} \text { greeted her. } \\
& \text { saw Mary. ??The } \operatorname{man}_{i} \text { greeted her. }
\end{aligned}
$$

(Guindon, 1985) proposed an alternative model of the attentional state involving a cache instead of a stack, and Walker (1996) argues that the cache model can account for all of the data that originally motivated the stack model and, in addition, explains the use of informationally redundant utterances. The cache model isn't yet specified in enough detail for all of its implications for the data discussed here to be clear, but it appears that some of the issues discussed in this paper would have to be addressed in a cache model as well, and that some of our conclusions would apply in a model of that type as well. In particular, these proposals are not very specific about whether the cache should count as a replacement of just the global focus component of G\&S's theory or of both local and global focus, and about what should go in the cache-Guindon seems to assume that it's discourse entities, whereas Walker also seems to allow for propositions and relational information. If the cache was intended as an alternative model of the global focus component, the data discussed here could be 
taken as an argument that what goes in the cache should be focus spaces with distinguished MSEs.

\section{Conclusions}

Our main intent in looking at long-distance pronominalisation was to make some of the aspects of the G\&S model of attentional state more precise, and to clarify its connection with earlier work by Sidner. The evidence we have presented suggests a main conclusion and a corollary. The main conclusion is that the uses of long-distance pronouns in our corpus can be explained as cases of reference to the MSE of a segment whose associated focus space is still on the stack. The corollary is that these examples can be accounted for within a G\&S-style model of discourse structure, provided that the theory is augmented by singling out some entities in focus spaces, and having these entities do some of the work done by Sidner's stack of discourse foci.

A concern with studies of this type is that notions such as 'most salient entity' are hard to define, and it's not obvious that two different researchers would necessarily agree on what is the MSE of a given sentence. Work on verifying whether the notion we are assuming can indeed be reliably identified is under way as part of the GNOME project.

\section{References}

H. H. Clark and C. J. Sengul. 1979. In search of referents for nouns and pronouns. Memory and Cognition, $7(1): 35-41$.

H. H. Clark. 1977. Bridging. In P. N. Johnson-Laird and P.C. Wason, editors, Thinking: Readings in Cognitive Science. Cambridge University Press.

B. A. Fox. 1987. Discourse Structure and Anaphora. Cambridge University Press, Cambridge, UK.

S. Garrod. 1993. Resolving pronouns and other anaphoric devices: The case for diversity in discourse processing. In C. Clifton, L. Frazier, and K. Rayner, editors, Perspectives in Sentence Processing. Lawrence Erlbaum.

P. Giouli. 1996. Topic chaining and discourse structure in task-oriented dialogues. Master's thesis, University of Edinburgh, Linguistics Department.

P. C. Gordon, B. J. Grosz, and L. A. Gillion. 1993. Pronouns, names, and the centering of attention in discourse. Cognitive Science, 17:311-348.

B. J. Grosz and C. L. Sidner. 1986. Attention, intention, and the structure of discourse. Conputational Linguistics, 12(3): 175-204.

B. J. Grosz, A. K. Joshi, and S. Weinstein. 1995. Centering: A framework for modelling the local coherence of discourse. Computational Linguistics, 21(2):202225.
B. J. Grosz. 1977. The Representation and Use of Focus in Dialogue Understanding. Ph.D. thesis, Stanford University.

R. Guindon. 1985. Anaphora resolution: Short-term memory and focusing. In Proc. of the 23rd Annual Meeting of the $A C L$, pp. 218-227.

U. Hahn and M. Strube. 1997. Centering in-the-large: Computing referential discourse segments. In Proc. of the 35th Annual Meeting of the ACL, pp. 104-111.

J. Hitzeman, C. Mellish, and J. Oberlander. 1997. Dynamic generation of museum web pages: The intelligent labelling explorer. Archives and Museum Informatics, 11:107-115.

J. R. Hobbs. 1978. Resolving pronoun references. Lingua, 44:311-338.

S.B. Hudson. 1988. The Structure of Discourse and Anaphor Resolution: The Discourse Center and the Roles of Nouns and Pronouns. Ph.D. thesis, University of Rochester.

W. C. Mann and S. A. Thompson. 1988. Rhetorical structure theory: Towards a functional theory of text organization. Text, 8(3):243-281.

J. D. Moore and C. L. Paris. 1993. Planning text for advisory dialogues: Capturing intentional and rhetorical information. Computational Linguistics, 19(4):651694, December.

M. Moser and J. Moore. 1996. Toward a synthesis of two accounts of discourse structure. Computational Linguistics, 22(3):409-419.

M. Poesio and R. Stevenson. 1998. Computational models of salience and psychological evidence. In preparation.

M. Poesio and R. Vieira. 1998. A corpus-based investigation of definite description use. Computational Linguistics. To appear.

M. Poesio, R. Vieira, and S. Teufel. 1997. Resolving bridging references in unrestricted text. In R. Mitkov, editor, Proc. of the ACL Workshop on Operational Factors in Robust Anaphora Resolution, pp. 1-6, Madrid.

M. Poesio, S. Schulte im Walde, and C. Brew. 1998. Lexical clustering and definite description interpretation. In Proc. of the AAAI Spring Symposium on Learning for Discourse, Stanford, CA, March.

M. Pollack, J. Hirschberg, and B. Webber. 1982. User participation in the reasoning process of expert system. In Proc. of AAAI-82, pp. 358-361.

C. L. Sidner. 1979. Towards a computational theory of definite anaphora comprehension in English discourse. Ph.D. thesis, MIT.

M. A. Walker. 1996. Limited attention and discourse structure. Computational Linguistics, 22(2):255-264.

B. L. Webber. 1991. Structure and ostension in the interpretation of discourse deixis. Language and Cognitive Processes, 6(2):107-135. 